\title{
Bracelets de pierre
}

\section{H. Camps-fabrer et B. Dudot}

\section{(2) OpenEdition}

Journals

Édition électronique

URL : http://journals.openedition.org/encyclopedieberbere/2650

DOI : 10.4000/encyclopedieberbere. 2650

ISSN : 2262-7197

\section{Éditeur}

Peeters Publishers

\section{Édition imprimée}

Date de publication : 1 septembre 1992

Pagination : 1603-1607

ISBN : 2-85744-581-4

ISSN : 1015-7344

\section{Référence électronique}

H. Camps-fabrer et B. Dudot, « Bracelets de pierre », Encyclopédie berbère [En ligne], 11 | 1992, document B103, mis en ligne le 01 juin 2012, consulté le 24 septembre 2020. URL : http:// journals.openedition.org/encyclopedieberbere/2650; DOI : https://doi.org/10.4000/ encyclopedieberbere. 2650

Ce document a été généré automatiquement le 24 septembre 2020.

(c) Tous droits réservés 


\title{
Bracelets de pierre
}

\author{
H. Camps-fabrer et B. Dudot
}

\section{Préhistoire (H. Camps-Fabrer)}

Des anneaux de pierre, généralement en stéatite, ont été trouvés dans de nombreux gisements néolithiques du Sahara central et méridional. Les rares bracelets ayant fait l'objet d'une étude sont ceux recueillis par la mission Augieras-Draper dans la région d'Asselar, ceux d'Amekni et ceux de la région de Foum el-Ahba et de l'erg Jmeya dans le Sahara malien. Les diamètres intérieurs de ces bracelets oscillent entre 50 et $70 \mathrm{~mm}$; les variations $\mathrm{du}$ diamètre extérieur sont considérables et n'ont pas grande signification car beaucoup d'anneaux brisés sont restés à l'état d'ébauche. L'un des rares exemplaires entiers est celui trouvé à Timelaïn, dans la Tefedest par J.-P. Maître. Les sections de ces bracelets sont en majorité triangulaires ou subtriangulaires, plus rarement subcirculaires.

2 A côté de ces bracelets dont les dimensions ne laissent aucun doute sur leur destination, il existe une série d'anneaux beaucoup plus épais. Tous ne sont pas des ébauches comme celles trouvées en abondance dans le gisement de l'Adrar Tiouiyne qui était certainement un atelier de fabrication de ces anneaux. Plusieurs peintures rupestres du style des Têtes rondes (dans la phase que A. Muzzolini a appelé les Martiens évolués), ces objets épais, volumineux sont de section semi-circulaire, et dans certaines représentations comme à Sefar et T-in Tazarift, ils ont l'aspect d'une sphère qui aurait été perforée pour permettre le passage du bras; mais rien ne permet d'affirmer que ces objets de parure étaient nécessairement en pierre. Il existe cependant des fragments très épais, achevés, ayant plus de $20 \mathrm{~mm}$ d'épaisseur et portant des stigmates qui prouvent qu'ils ont été portés ou utilisés; il est vrai que de telles boules, perforées plus ou moins largement, pouvaient lester des bâtons à fouir comme celui représenté dans une peinture de style bovidien de Sefar.

Relativement fréquents au Sahara central et méridional, les anneaux de pierre semblent inconnus ou du moins très rares dans le Nord et au Maghreb, alors que les boules perforées ne sont pas exceptionnelles dans ces régions. Les anneaux de bras ou 
de poignet que portent sur les gravures rupestres les personnages de style naturaliste (Gada el Kharrouba, Aïn Naga...) ne sont indiqués que par un trait simple ou deux incisions très rapprochées.

\section{Bracelets en pierre (chez les Touaregs) (B. Dudot)}

4 Les anneaux de bras de formes diverses que portent les Touaregs - et parfois aussi, les Peuls Bororo de l'Azawak - au-dessus du coude d'un ou des deux bras, sont faits d'une stéatite provenant du massif de l'Aïr. Les Touaregs de la Boucle du Niger portent aussi des anneaux, faits d'une sorte de marbre provenant des Monts Hombori, selon Henri Lhote.

Dans l'Aïr, la matière première est donc une stéatite de couleur gris-verdâtre, ayant parfois au toucher l'onctuosité du savon; cette roche ne se trouverait que près d'un petit massif, l'Adrar Aré, situé à l'est des Monts Bagzane, en bordure du désert du Ténéré. La pierre est extraite du sol, sous le sable dunaire. Ce lieu d'extraction est situé à deux jours de chameau du village de Tabelot, soit à peu près une cinquantaine de kilomètres.

Un certain nombre de forgerons fabriquant ces anneaux sont originaires des environs d'Abardak et de Tabelot, au pied des Bagzane. Les hommes demeurent une semaine environ sur le lieu d'extraction avec des vivres et de l'eau pour tirer la pierre du sol et l'apprêter. Après avoir chargé leurs bêtes, ils prennent la direction d'Agadez. A chaque halte, pendant la préparation du repas, chacun travaille la pierre. A Agadez, leur arrêt se prolonge jusqu'à l'achèvement de la fabrication et l'épuisement de la matière première. Un certain nombre d'anneaux est acheté par des revendeurs sur le marché de la ville; mais souvent les artisans prennent la piste de Dakoro, marché important à la jonction des zones nomades et sédentaire, et où les anneaux de bras se vendent à un prix supérieur. 

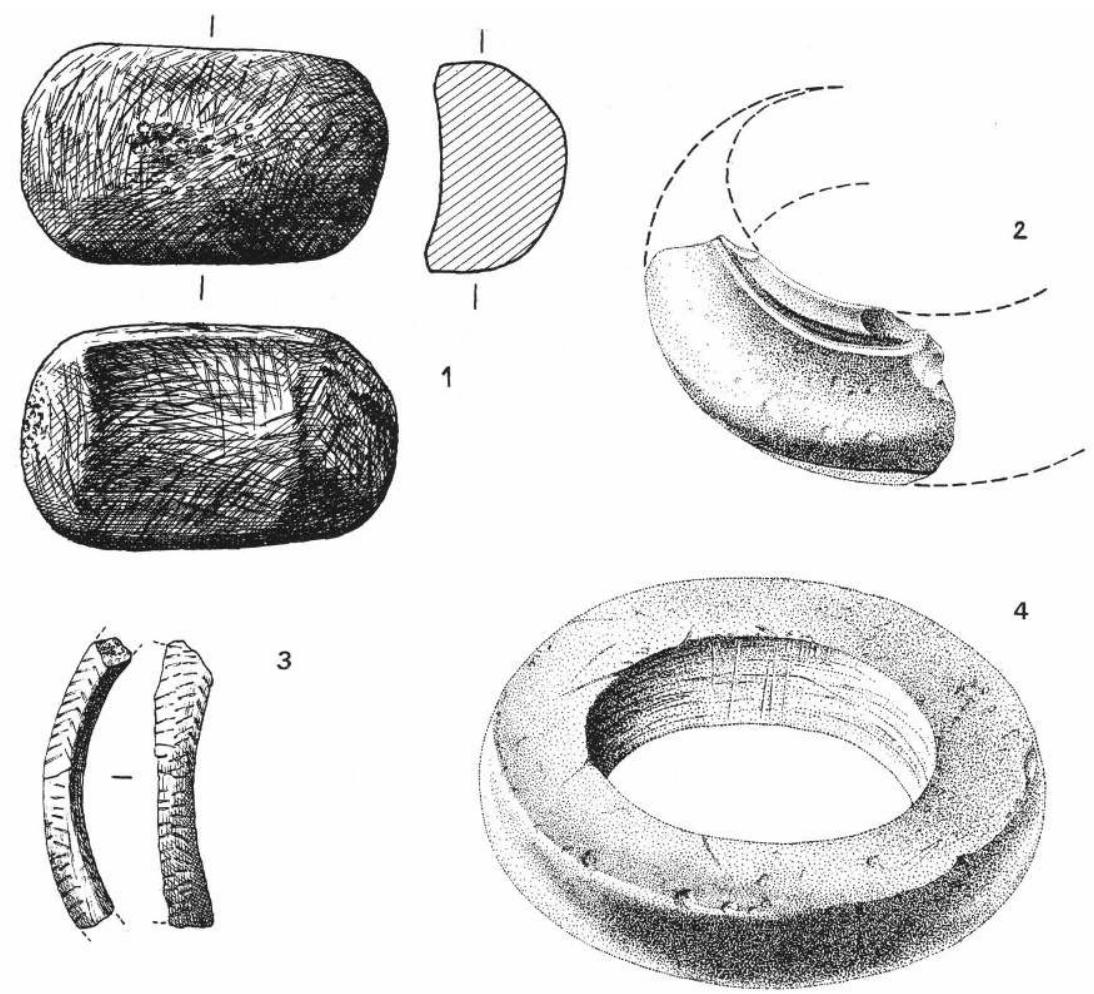

1 et 3 d'Amekni, 2 d'Ouhet, 4 de Timelaïn

(dessins Y. Assié)

\section{Fabrication des bracelets}

7 La roche schisteuse, extraite $\mathrm{du}$ sol, est débitée en plaques grossièrement rectangulaires (environ $25 \mathrm{~cm} \times 12 \mathrm{~cm} \times 3-5 \mathrm{~cm}$ ). Le type d'anneau fabriqué dépendra de l'épaisseur de la plaque; ainsi une pierre aux dimensions indiquées plus haut permet d'obtenir quatre anneaux du type asket ou deux anneaux du type agosrer.

La plaque rectangulaire est divisée en deux morceaux sensiblement carrés ; puis chacun est arrondi à coups d'herminette et creusé sur les deux faces d'un sillon circulaire ; peu à peu les deux sillons finissent par se rejoindre et la partie centrale se détache : on obtient ainsi une ébauche appelée maraba ; celle-ci est sciée en deux, dans le sens du plan circulaire, quand on veut obtenir deux anneaux asket; il n'est pas scié quand il s'agit d'obtenir des modèles plus épais, du type agosrer, ou abambey ou imi n'ezennou.

9 L'artisan donne alors l'ébauche de la forme désirée avec une herminette à lame plus étroite. On désigne les anneaux en général, ainsi que la pierre dont ils sont tirés, par le nom d'iwuki; mais chaque type d'anneau porte un nom propre: asket, abambey, agosrer, imi n'ezennou, ebelbal. Les anneaux sont ensuite polis à la lime. Enfin ils sont enduis de matière grasse, huile ou beurre, puis exposés à la fumée et à la flamme d'un feu de paille durant quelques instants ; frottés ensuite avec un chiffon, ils apparaissent recouverts d'un beau poli noir. La sueur, le frottement sur la peau de celui qui le portera, et aussi l'indigo des vêtements, donneront à l'anneau sa patine définitive. 
10 grands, destinés aux très jeunes garçons. De cette pierre, ils font aussi des sortes de coupelles ressemblant à des bols européens, mais plus petites et sans pied, dans lesquelles les femmes touarègues conservent du beurre pour graisser leurs cheveux.

Achèvement à la lime d'un bracelet de pierre

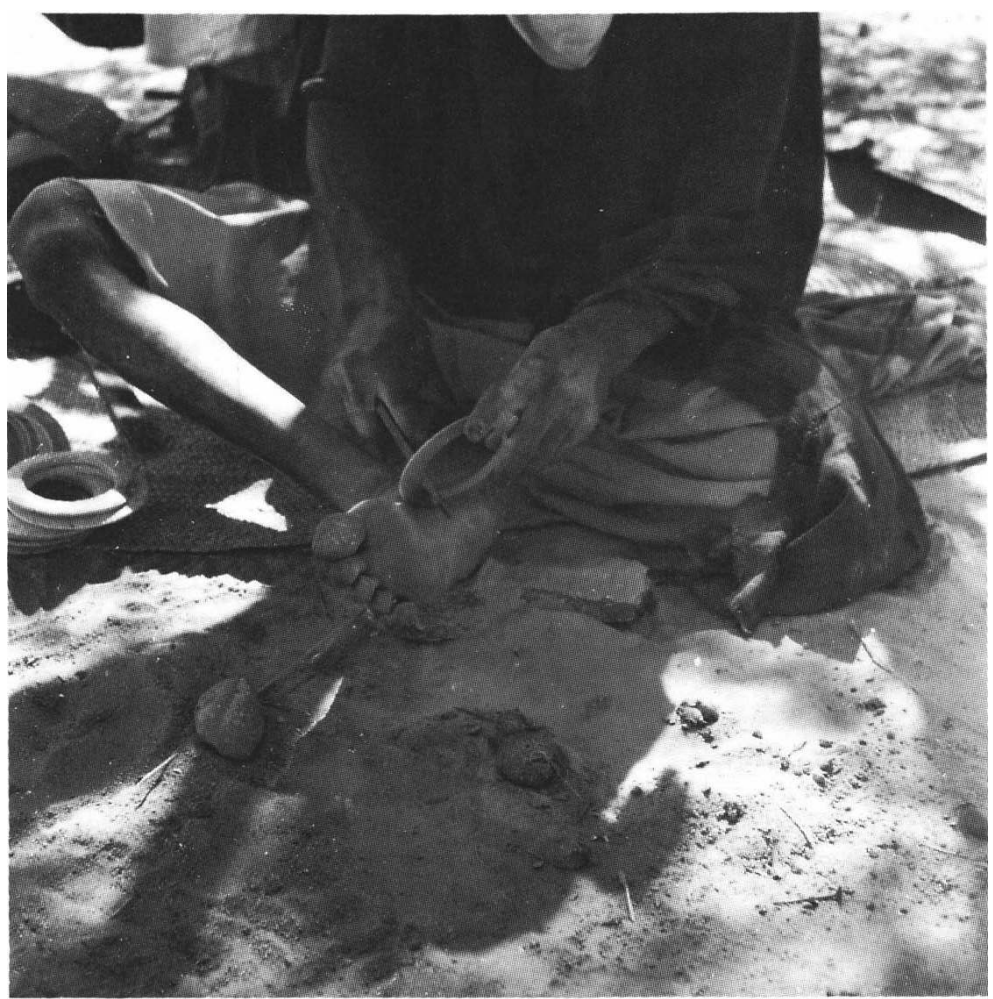

(photo B. Dudot).

11 Depuis quelques années, certains forgerons d'Agadez fabriquent avec cette pierre des cendriers et des porte-couteaux en forme de margouillat, et proposent ces objets aux touristes.

12 Les outils utilisés pour le travail des anneaux sont en nombre réduit :

- une grosse herminette servant à dégrossir et à tailler les pierres et les anneaux bruts ; - une herminette plus petite destinée à donner la forme propre à chaque type d'anneau ;

- une lame de scie assez rudimentaire, fabriquée dans un morceau de tôle de fer, tendue sur un support en fer, à l'imitation des scies à métaux européennes ;

- un jeu de limes d'importation, pour le polissage des anneaux.

13 L'habileté manuelle de l'artisan compense la grossièreté de l'outillage ; un bon ouvrier peut confectionner une trentaine d'anneaux par jour, me disait mon informateur qui était spécialisé dans ce travail ; il ajoutait qu'il lui arrive d'en casser quatre à cinq au cours de la fabrication, notamment pendant la taille. 
Principaux types de bracelets en pierre des Touaregs de la région d'Agadez

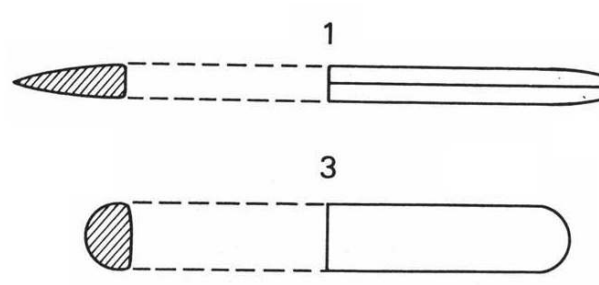

2

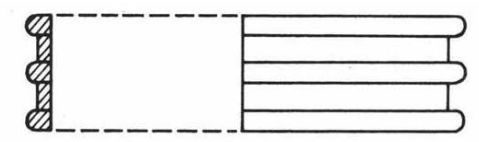

4

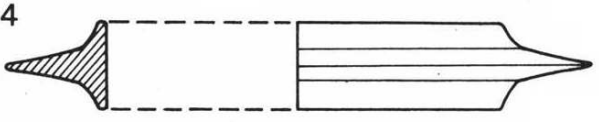

1. Asket, 2. Imi n-Ezenou, 3. Abambey, 4. Agosrer

(Dessin Y. Assié).

\section{Le port des anneaux de pierre chez les Touaregs}

Dans son ouvrage sur les Touaregs, Henri Lhote attribue aux anneaux de pierre un caractère à la fois ornemental et magique, contredisant Duveyrier qui les a décrits comme étant des armes. Lhote écrit : « ... l'anneau de pierre est tellement fragile qu'on ne saurait lui faire subir une forte pression sans le faire éclater, ce qui risquerait de blesser gravement l'homme qui le porte ». Il ajoute plus loin que «si l'anneau de bras ne doit pas être considéré comme une arme, il a certainement un caractère magique qui a pour but de rendre le bras plus fort dans son action. »

M.-F. Nicolas, à propos des Ioullemmeden de l'Est, affirme lui aussi que c'est une arme ; il écrit : « L'iwuki est un ornement et une arme ; comprimant le biceps à la base pendant l'effort, il donne, disent les nomades Twareg, un surcroît de vigueur; dans la lutte athlétique entre deux hommes, la prise de tête entre le coude et la hanche comprime le cou ou la tempe de l'adversaire et le met hors de combat. "

Une opinion répandue chez les Européens tendrait à faire croire que ces anneaux étaient destinés à amortir les coups de takouba dans les combats ; quand on a manipulé cette pierre et qu'on a vu par expérience le peu de résistance qu'offrent ces anneaux à des chocs violents, on se rend compte que cette opinion est irrecevable. Je pense, par ailleurs, que, s'il en était ainsi, les Touaregs qui, encore aujourd'hui, ne sont pas avares de coups de takouba lors de querelles avec les Peuls autour des puits, en feraient état ; mais l'explication qu'ils donnent, au Niger, rejoint celle de H. Lhote.

Les Touaregs du nord de Tahoua m'avaient confirmé ce caractère magique à plusieurs reprises. A Agadez et dans l'Aïr, les porteurs d'anneaux interrogés m'ont tous dit que leur bracelet était un ornement et surtout une protection contre toutes paroles malveillantes; un forgeron d'Agadès-Toudou expliquait cela ainsi: «Lorsque des injures, des paroles blessantes étaient adressés à un homme porteur d'un anneau, l'influence malfaisante des mots atteignait l'anneau et non la personne: la pierre protégeait l'homme, mais sous le choc maléfique, se brisait. »

8 Me parlant des différents types d'anneaux, ce même forgeron expliquait que beaucoup de Touaregs blancs préféraient porter les modèles asket, agosrer, imi n'ezennou, laissant le type abambey aux bouzou captifs ou anciens captifs de race noire. En pratique, les types asket et agosrer sont les modèles les plus répandus chez tous les Touaregs.

Ces anneaux sont souvent agrémentés ou surchargés d'amulettes de cuir, de formes diverses, qui augmentent encore le pouvoir protecteur magique de la pierre. Il semblerait bien que la vertu magique réside dans la matière elle-même: ce schiste 
tendre, réduit en poudre et saupoudré sur les plaies des hommes et des chameaux, aide à guérir les blessures : c'est le "sulfamide » local.

Souvent les anneaux portent des inscriptions en tifinar : elles sont parfois tracées par des femmes ou des jeunes filles et ont un caractère sentimental, mais ce n'est pas toujours le cas.

En conclusion, je citerai une anecdote qui m'a été contée à Agadez, montrant l'importance qu'attachaient les Touaregs à ces anneaux de bras: Durant la guerre qui opposa les troupes françaises aux Touaregs de Kaosen, les anneaux de bras de l'Aïr parvenaient très difficilement chez les Ioullemmeden de l'Azawak. Si d'aventure un Touareg de l'Aïr, porteur d'anneaux de bras, était surpris, isolé, par les «Tegareygarey » (nom donné aux Touaregs de l'Azawak par ceux de l'Aïr) et s'il était vaincu dans le combat qui s'ensuivait, ses adversaires, dans leur hâte de s'emparer des anneaux, n'hésitaient pas à couper le bras de l'homme terrassé quand ils ne pouvaient pas retirer facilement ceux-ci.

\section{Les noms des types d'anneaux}

Asket : voudrait dire littéralement : couper en tranche. En effet, cet anneau est obtenu en « coupant en tranche » l'anneau de pierre brut.

Abambey: ce mot désigne un rouleau de bandes tressées en feuilles de palmier doum, bandes dont les femmes se servent pour confectionner des nattes. L'anneau de ce nom ferait-il penser à ces rouleaux ?

Agosrer : voudrait dire : « le plus joli des anneaux », celui qui plaît le plus. C'est en effet l'un des plus répandus.

Imi n'ezennou : mot composé de imi, la bouche, et ezennou, le fruit de la gourde, variété de calebasse* dont on fait des récipients, après en avoir vidé la pulpe et les graines desséchées; le nom haoussa de cet anneau est : baaki n'tulu (ce qui veut dire : la bouche du canari, de baaki, la bouche et tulu, grand vase à large ouverture contenant la réserve d'eau potable). L'anneau fait donc penser au col plus ou moins large d'un récipient à eau (léger et facile à porter comme la calebasse pour le nomade, ou de grande capacité et volumineux comme les grands canaris des sédentaires).

\section{BIBLIOGRAPHIE}

\section{H. CAMPS-FABRER}

Monod Th., « Découverte d'un homme fossile. Quelques observations sur les habitants actuels et la Préhistoire ». D’Algérie au Sénégal. Mission Draper-Augieras, 1927-1928, Paris, Challamel, 1931, p. 252-284.

Camps-Fabrer H., Parures des temps préhistoriques en Afrique du Nord, Alger, Impr. officielle, 1961.

Camps G. éd., Amekni, Néolithique ancien du Hoggar, Mém. du CRAPE, X, Paris, A.M.G., 1969, p. 153. 
Maître T.-P., Contribution à la préhistoire de l'Ahaggar. I. La Téfedest centrale, Mém. du CRAPE, XVII, Paris, A.M.G., 1971.

Petit-Maître N. et Riser J. éd., Sahara ou Sahel. Quaternaire récent du Bassin de Taoudenni (Mali), Paris, CNRS, 1983. « Parures et engins de pêche » par H. Camps-Fabrer, p. 367-409.

B. DUDOT

Lhote H., Les Touaregs du Hoggar, Paris, Payot, 1944.

Collections ethnographiques, Musée d'Ethnographie et de Préhistoire du Bardo, Alger, Paris, A.M.G. 1959, pl. V, légende de M. Gast.

Arkell A., « Autour des anneaux de pierre polie », Notes africaines, Dakar, octobre 1959, p. 84-99.

INDEX

Mots-clés : Artisanat, Ethnographie, Sahel, Technologie 